\title{
Small Heterocyclic Molecule as Multistate Transistor : A Quantum Many-body Approach
}

\author{
Dibyajyoti Ghosh, ${ }^{1}$ Prakash Parida, ${ }^{2}$ and Swapan K. Pati ${ }^{3, *}$ \\ ${ }^{1}$ Chemistry and Physics of Materials Unit, \\ JNCASR, Bangalore-560064, Karnataka, India \\ ${ }^{2}$ Department of Physical Sciences,, Central University of Punjab, Bathinda, Punjab \\ ${ }^{3}$ Theoretical Sciences Unit, JNCASR, \\ Bangalore-560064, Karnataka, India
}

\begin{abstract}
Weakly coupled molecular junctions are quite active and important field of research as they exhibit various non-linear transport phenomena. We have investigated the carrier transport through weakly coupled $\mathrm{B}_{2} \mathrm{C}_{2} \mathrm{~N}_{2} \mathrm{H}_{6}$ molecules using quantum many body approach coupled with kinetic (master) equations. Various types of non-linear current voltage characteristics, such as, negative differential conductance (NDC), rectifications, Coulomb staircase, which are hallmark of multistate transport devices, have been obtained. Source-drain voltage induces changes in the occupation probabilities of low-lying many body states depending on the nature of the carrier transport. The nature of the carrier transport directly controls the net current flowing through the molecular junctions. We further investigated the effect of different kinds of perturbations such as application of gate voltage and magnetic field perpendicular to the direction of the molecule upon the carrierflow through various molecular bridges. Interestingly, we find that depending on the strength of the applied perturbing field, several phenomena, such as, switching off of the current, suppression of NDC etc. appears in the device. Fundamentally, this applied perturbation modifies both the site charge density as well as occupation probabilities of transport active channels, resulting in significant alteration in the transport behavior of the molecular junction.
\end{abstract}




\section{INTRODUCTION}

Molecular electronics, the investigation of the electronic properties of circuits which are composed of individual molecules, have gained a huge research attention in past the few decades $\frac{112}{}$. Appearance of efficient switching ${ }^{3}$, negative differential conductance (NDC) ${ }^{4}$, rectification ${ }^{5}$, spin-transport ${ }^{6}$, spin-filtration ${ }^{7}$, thermoelectric effect ${ }^{8}$ in various molecular junctions evidently shows the potential of these nano-devices to be used as alternative of conventional silicon-based semiconductor electronics. Among all these fascinating properties, particularly, rectification and NDC characteristics have been explored quite thoroughly from the very beginning of this field of study. Efficient rectification and / or NDC has been observed in a number of molecules attached strongly to the bulk electrodes. This is attributed mainly to (1) asymmetric nature of the molecule (donor-acceptor molecules) ${ }^{5}(2)$ different electrode-molecule coupling strengths (asymmetric anchoring groups or electrodes) ${ }^{5}$ (3) different spatial potential profiles ${ }^{4}$ etc. Although these phenomena mainly appear for covalently bonded molecule-electrode systems (i.e. coherent tunneling regime), recent experimental and theoretical investigations demonstrated the same for weakly coupled molecular junctions(sequential tunneling regime). ${ }^{49}$. Small molecules (such as benzene) and double quantum dots (like GaAs-based QDs) which are coupled weakly to metallic electrodes have shown prominent rectification and NDC properties, due to various factors, such as, internal charge transfer, intrinsic molecular asymmetries, interference effects, Pauli spin-blocking etc. $4[10,12$.

Recently, there is a huge surge in interest in the modulation of electron transport through these molecular junctions by including different kinds of optical, magnetic and electronic perturbations. Particularly, it has been demonstrated that depending upon various conditions, the carrier transport through weakly coupled molecule-electrode systems can become very sensitive towards the applied external magnetic fields. The magnetic field induced tuning of current may arise due to several factors, such as, tuning of interfering electronic degenerate states, modulation of the sharp transmission resonances etc.

Theoretical modeling of these weakly coupled devices show that coherent non-equilibrium Greens function (NEGF) ${ }^{13[14}$ formalism coupled with self-consistent field (SCF) approach is not adequate to reproduce experimental findings even at the qualitative level. Unlike in the case of strongly coupled systems, charging energies of molecules/QDs in these devices are 
much higher than electrode coupling and plays a major role (in what?) $\underline{15 \mid 16}$. Consequently, these devices remain in Coulomb blockade (CB) regime where integral charge transfer dominates the electron transport through molecular junctions. To describe the molecular transport in the $\mathrm{CB}$ regime the quantum master/rate equation approach is widely used $\frac{17 \mid 18}{18}$. This formalism efficiently describes electron transport through many-body eigenstates of molecular systems. Since charging energy is much higher than the molecule/dot electrode coupling, in the weak coupling limit, we do not explicitly consider the electrode or its coupling with the molecule/dot device, in the kinetic equation method. We discuss the formalism in detail in Models and Methods section of the paper.

Using this approach, Hettler et. al. have demonstrated the large NDC behavior in weakly coupled benzene-based molecular junctions 10 . They proposed that under a finite bias, the radiative relaxation of electrons populate a particular many-body state which blocks the transport of current, resulting in the NDC behavior. Darau et al. revisited the same system with generalized master equation approach, where strong interference effect appears to be the reason for the observed NDC behavior ${ }^{19}$.

Apart from molecules, donor-acceptor QDs have also been investigated thoroughly for their various non-linear transport characteristics in weak coupling regime. Muralidharan et al have demonstrated the criterion to find NDC in these double QDs in terms of transition rates for populating and depopulating the transport-active many-body states $\frac{16}{}$. Song et al have observed rectification in I-V characteristics for weakly coupled spatially separated donor-acceptor systems ${ }^{20}$. Difference in coupling strengths of these sites to the electrodes results in the rectification effect in these molecular junctions. Parida et al. have used kinetic equation approach to investigate transport characteristics in donor-acceptor double QD systems 11 . They propose that the increased population of the non-conductive triplet state with increase in bias voltage and consequent reduction in the current transport leads to a prominent NDC feature.

In this paper, we consider a heterocyclic benzene i.e. $\mathrm{B}_{2} \mathrm{C}_{2} \mathrm{~N}_{2} \mathrm{H}_{6}$ (see the inset of Fig. 1) as the molecular bridge which is weakly coupled to the metallic electrodes on either side. Thus, the molecular junction is effective in Coulomb blockade regime. Here, B, C and N sites act as acceptor, bridge and donor (from the electron point of view), respectively due to their intrinsic chemical nature. This molecule can efficiently be modeled as two identical donor-bridge-acceptor half-rings (B-C-N), connected to each other in end-on manner by 
covalent bonds. We construct the interacting isolated molecular Hamiltonian considering only the localized $2 \mathrm{p}_{\mathrm{z}}$ orbitals of $\mathrm{B}, \mathrm{C}$ and $\mathrm{N}$ atoms ${ }^{10 \mid 19}$ since the $\sigma$ orbitals are at a much higher energy scale, as followed in earlier works. Depending upon the pair of sites involved in electrode-molecule coupling, we find various non-linear current-voltage characteristics, such as, prominent NDC, strong rectification, Coulomb staircase, in this two-terminal molecular device. We analyzed the bias-dependent probability of many-body states by looking at the charge distributions at all atomic sites for each of these low-lying states (also called transport-active channels.) We further looked into the carrier transport in these weakly coupled systems by applying perpendicular magnetic field of various strength. The current transport appears to be quite sensitive to the applied magnetic field. The effect of magnetic field over transport strongly depends on various factors such as (1) strength of the magnetic field and (2) relative position of electrodes. The change in atomic charge distributions as well as occupation probabilities of low-lying states upon the application of magnetic fields hugely alters the current-voltage characteristics of these molecular devices.

\section{MODEL AND METHODS}

We undertake the quantum master-equation approach to explore transport characteris-

tics of $\mathrm{B}_{2} \mathrm{C}_{2} \mathrm{~N}_{2} \mathrm{H}_{6}$ (see the schematic in the inset of Fig. 11) in the sequential tunneling i.e. Coulomb blockade regime. This approach formulates the carrier transport through a correlated system, having many-body eigenstates. In this present approach, the occupation probabilities of many-body states are calculated from their corresponding wave functions. Note that, in the present study, we neglect the off-diagonal coherence while solving the rate equation.

We model $\mathrm{B}_{2} \mathrm{C}_{2} \mathrm{~N}_{2} \mathrm{H}_{6}$ molecule by taking 6 electrons in 6 sites within Hubbard model. Taking into account the on-site electron-electron interactions and hopping between nearest neighbor sites, we write down the most general form of the Hamiltonian as follows,

$$
H=\sum_{i=1, \sigma}^{6}\left(\epsilon_{i}-e V_{g}\right) a_{i \sigma}^{\dagger} a_{i \sigma}+\sum_{i \sigma}-t\left(a_{i \sigma}^{\dagger} a_{i+1, \sigma}+\text { h.c. }\right)+U \sum_{i=1}^{N} n_{i \uparrow} n_{i \downarrow}
$$

where $\mathrm{t}$ is hopping strength between nearest neighbor sites, $\epsilon_{i}$ is the on-site energy for 
different atomic sites, $U$ is the Hubbard interaction term and $V_{g}$ represents the external gate bias. We consider only nearest neighbor hopping and have taken equal hopping strength $(2.4 \mathrm{eV})$ for all bonds, i.e., B-C, C-N and B- $\mathrm{N}^{10121}$. Here, we neglect the small differences among the hopping parameters which may appear due to different chemical nature of $\mathrm{C}, \mathrm{B}$ and $\mathrm{N}$ atoms. For Hubbard parameters, we obtain Hubbard on-site energy as the difference between 1st ionization potential and electron affinity of each of the atomic species. Thus, the Hubbard on-site parameters are evaluated to be $8.02 \mathrm{eV}, 9.67 \mathrm{eV}$ and $14.46 \mathrm{eV}$ for $\mathrm{B}, \mathrm{C}$ and $\mathrm{N}$, respectively 22 . The site-energy term in the Hamiltonian corresponds to negative of first ionization potential for each atomic species. And these turn out to be $-8.30 \mathrm{eV},-11.26$ $\mathrm{eV}$ and $-14.53 \mathrm{eV}$ for $\mathrm{B}, \mathrm{C}$ and $\mathrm{N}$, respectively.

To compute the transport properties in the sequential tunneling limit, we diagonalize the Hubbard Hamiltonian, $H$. Diagonalization of $H$ gives many-body eigenstates $\mid s>$ with the corresponding eigen energies, $E_{s}$. We compute the occupation probabilities $P_{s}$, through the master equation approach, in the steady state of the system. The transition rate, $W_{s^{\prime} \rightarrow s}$, from many-body state, $s^{\prime}$ of the molecule with $N$ electrons to a state $s$ with $(N-1)$ or $(N+1)$ electrons, is calculated up to linear order in $\Gamma$, where $\Gamma$ is the bare electron tunneling rate between the molecule and the left/right electrode. Using the Fermi's golden rule the transition rate can be written as follows,

$$
\begin{gathered}
W_{s^{\prime} \rightarrow s}^{L+}=\Gamma f_{L}\left(E_{s}-E_{s}^{\prime}\right) \sum_{\sigma}|<s| a_{1 \sigma}^{\dagger}\left|s^{\prime}>\right|^{2} \\
W_{s^{\prime} \rightarrow s}^{R+}=\Gamma f_{R}\left(E_{s}-E_{s}^{\prime}\right) \sum_{\sigma}|<s| a_{N \sigma}^{\dagger}\left|s^{\prime}>\right|^{2}
\end{gathered}
$$

The corresponding equation for $W_{s \rightarrow s^{\prime}}^{L-}$ and $W_{s \rightarrow s^{\prime}}^{R-}$ are formulated by replacing $f_{L, R}\left(E_{s}-E_{s}^{\prime}\right)$ by $\left(1-f_{L, R}\left(E_{s}-E_{s}^{\prime}\right)\right)$, where $f_{L / R}$ is the Fermi function for left/right electrode. Here, $+/$ - represents the creation/annihilation of an electron inside the molecule due to electron movement from/to left $(L)$ or right $(R)$ electrodes. $C_{1 \sigma}^{\dagger}$ and $C_{N \sigma}^{\dagger}$ are the creation operators for electrons with spin, $\sigma$, at the $1^{\text {st }}$ and $N^{\text {th }}$ lattice sites, respectively. We also have assumed that the creation and annihilation happens only at the sites which are directly connected to the electrodes. The total transition rate is obtained as, $W_{s \rightarrow s^{\prime}}=W_{s \rightarrow s^{\prime}}^{L+}+W_{s \rightarrow s^{\prime}}^{R+}+W_{s \rightarrow s^{\prime}}^{L-}+$ 
$W_{s \rightarrow s^{\prime}}^{R-}$. Now. the non-equilibrium probability, $P_{s}$, of occurrence of each many-body state, $s$, can be represented by the rate equation,

$$
\dot{P}_{s}=\sum_{s^{\prime}}\left(W_{s^{\prime} \rightarrow s} P_{s^{\prime}}-W_{s \rightarrow s^{\prime}} P_{s}\right)
$$

At the steady state, the population of the different many-body states $\left(P_{s}\right)$ can be found by solving the above rate equation ( Eq. (3) ). Thus, the Eq. (3) becomes

$$
\dot{P}_{s}=\sum_{s^{\prime}}\left(W_{s^{\prime} \rightarrow s} P_{s^{\prime}}-W_{s \rightarrow s^{\prime}} P_{s}\right)=0 .
$$

The Eq. (4) can be written in an expanded form that results in a homogeneous linear system $(\mathrm{AX}=0)$ of the size of the many-body space. Since $\mathrm{AX}=0$ can not be solved, we make use of $\sum_{\mathrm{s}} \mathrm{P}_{\mathrm{s}}=1$ to eliminate one row/column, thus reformulating the eigenvector problem into an inhomogeneous linear system $(\mathrm{AX}=\mathrm{B})$, which can be solved using well-known linear algebraic methods 23 . Thereafter, the current in the left and right electrodes is calculated by the following formula,

$$
I_{\alpha}=\frac{e}{\hbar} \sum_{s, s^{\prime}}\left(W_{s^{\prime} \rightarrow s}^{\alpha+} P_{s^{\prime}}-W_{s \rightarrow s^{\prime}}^{\alpha-} P_{s}\right)
$$

where $\alpha=L / R$. Note that at steady state, the current at two terminals is same, i.e. $I_{L}(t)=I_{R}(t)=I(t)$.

Further we also study the carrier transport through these molecular junctions in the presence of perpendicular magnetic fields. We consider the effect of applied magnetic field $(B)$ by modifying the hopping parameter, $t_{i j}$, such that it acquires (?)/includes(?) a Peierl's phase, as discussed in earlier works. $\frac{2425}{25}$ Thus, $t_{i j}$ modifies as $t_{i j} e^{\frac{2 \pi i \delta \phi}{\phi_{0}}}$, where $\phi_{0}=h / e$ is the quantum of magnetic flux, and $\delta \phi$ is the Peierl's phase given by,

$$
\delta \phi=\int_{i}^{j} \mathbf{A} \cdot d \mathbf{l}
$$

where $\mathbf{A}$ is the vector potential created by the perpendicular B-field, $\mathbf{B}=(0,|\mathbf{B x}|, 0)$. Magnetic flux, $\phi$, correspdoning to this magnetic field is $\phi=\mathbf{B} \cdot A_{s}$, where $A_{s}$ is the area of the hexagonal ring i.e. $3 \sqrt{ } 3 a^{2} / 2$ with a as the bond length (or radius of the ring???). 
We investigate the transport characteristics for a donor-bridge-acceptor molecular circuit in weak-coupling regime. We simulate the molecular junction at room-temperature, $\mathrm{T}=$ $300 \mathrm{~K}$ and consider $\Gamma$, the molecule-electrode coupling strength, to be $0.25 \mathrm{meV}$. We consider symmetric electrode-molecule coupling for the presently studied junctions. In our case, asymmetry in the system is inherent and appears due to the different chemical nature of constituent atoms. We apply the exact diagonalization (ED) method to diagonalize the 6-site Hamiltonian, as described in equation Eq. (1), with a total number of basis of $6^{4}$ i.e. 4096.

Several previous investigations established the fact that asymmetric coupling strengths for left and rig

\section{RESULTS AND DISCUSSION}

We first investigate the stability of different charge states of the molecule under applied gate voltage $\left(V_{G}\right)$, in the absence of magnetic field. We have given the number of electrons in the molecule with lowest energy under various gate bias values, keeping source-drain bias $\left(V_{S D}\right)$ zero in Fig. 1. (NOT CLEAR) As can be seen in Fig. 1, the six electron molecular state has widest plateau, indicating that this charge state is the ground state of the molecule. Consequently, we choose the Fermi energy of the device such that molecule remains in its ground state.

As the molecule is cyclic in nature, we can connect the electrodes to it in different possible conformations such as ortho, meta or para positions. We further fix the Fermi energy in such a way that small $V_{S D}$ bias can transform the molecule from 6 electron ground state to 7 electron ground state i.e. anionic state with one more electron. Note that, in this paper, we are investigating the I-V characteristics of the device, considering only neutral or anionic molecule. CHANGE THIS SENTENCE The I-V characteristics of three different connectivities are given in Fig. 2, From Fig. 2, it is evident that I-V characteristic of these junctions is highly sensitive towards the chemical nature of molecular site to which the

electrode is connected. We will discuss the different kinds of non-linear transport behaviours such as negative differential conductance (NDC), rectification and staircase, in next sections. 


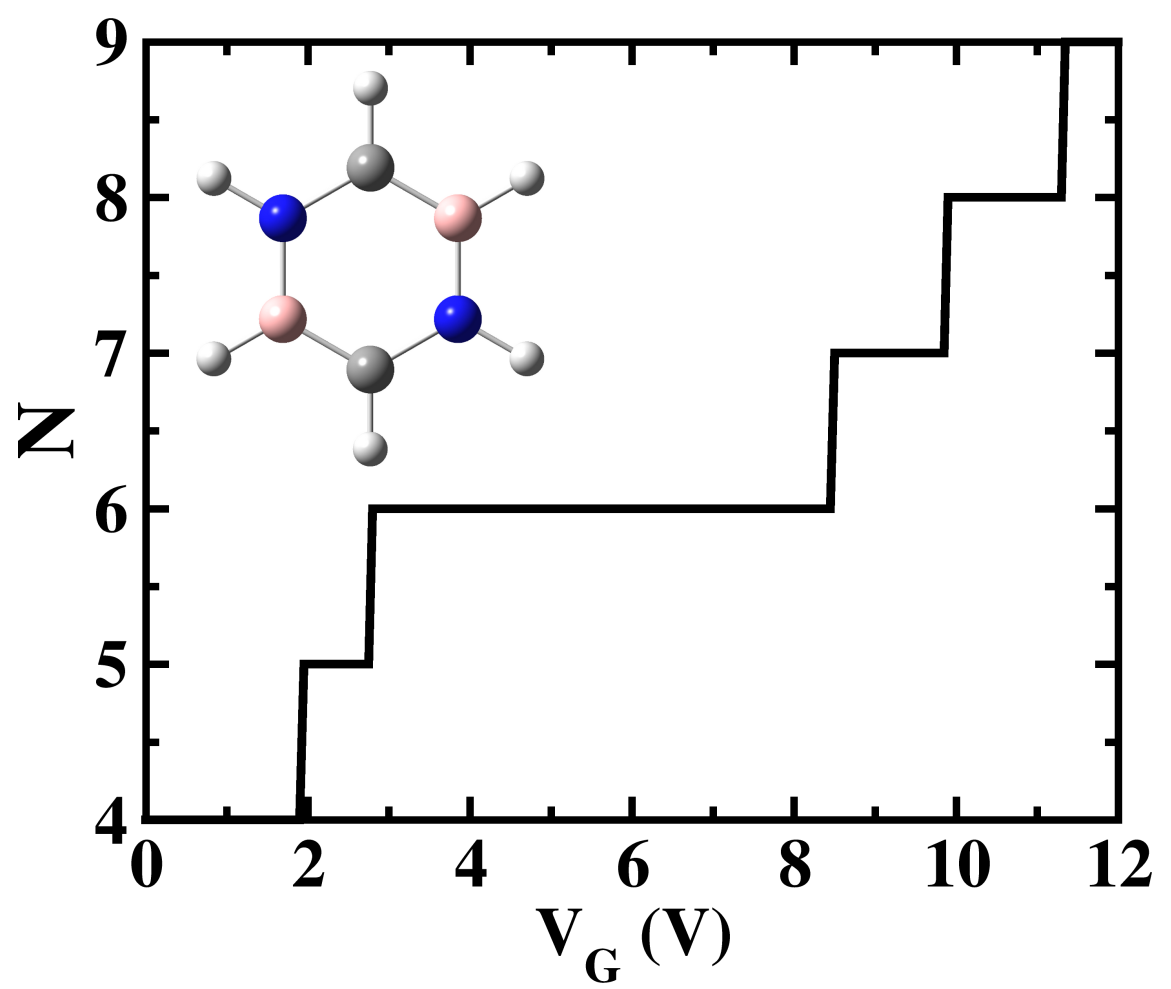

FIG. 1. The number of electrons $(\mathrm{N})$ in the molecule with variation of gate voltage $\left(V_{G}\right)$ of the device. We have not included the spin sector in the present plot. In the inset, a schematic representation of a $\mathrm{B}_{2} \mathrm{C}_{2} \mathrm{~N}_{2} \mathrm{H}_{6}$ molecule is given. Hydrogen, boron, carbon and nitrogen are represented by white, lime, gray and blue colored spheres.

\section{A. Negative Differential Conductance}

Firstly, we consider the molecular bridge, (particular device) where both para-positioned $\mathrm{B}$ atoms are weakly connected to the electrodes. Note that, the I-V characteristic of this device is identical at both the positive and negative bias, but with an opposite sign. i.e. $\mathrm{I}\left(V_{S D}\right)=\mathrm{I}\left(-V_{S D}\right)$. It is quite obvious, since both the geometry and strength of moleculeelectrode coupling are symmetric in nature. This symmetric nature of I-V characteristic has already been observed for benzene and double quantum dot systems. 10116 Taking the advantage of this symmetry, here we discuss the I-V characteristics, focusing only on positive bias regime. As shown in the inset of Fig. 3 (a), at low bias i.e. $V_{S D}<1.0 \mathrm{~V}$, the current appears to be very small, of the order of pA only. However, most important feature of this nano-junction appears at the bias range of $1.0<V_{S D}<2.2 \mathrm{~V}$, where current rises sharply and then suddenly decreases with the increase in $V_{S D}$, exhibiting a strong NDC behavior (see Fig. $3(\mathrm{a})$ ). With further increase in bias, i.e. $V_{S D}>2.80 \mathrm{~V}$, the transport current 


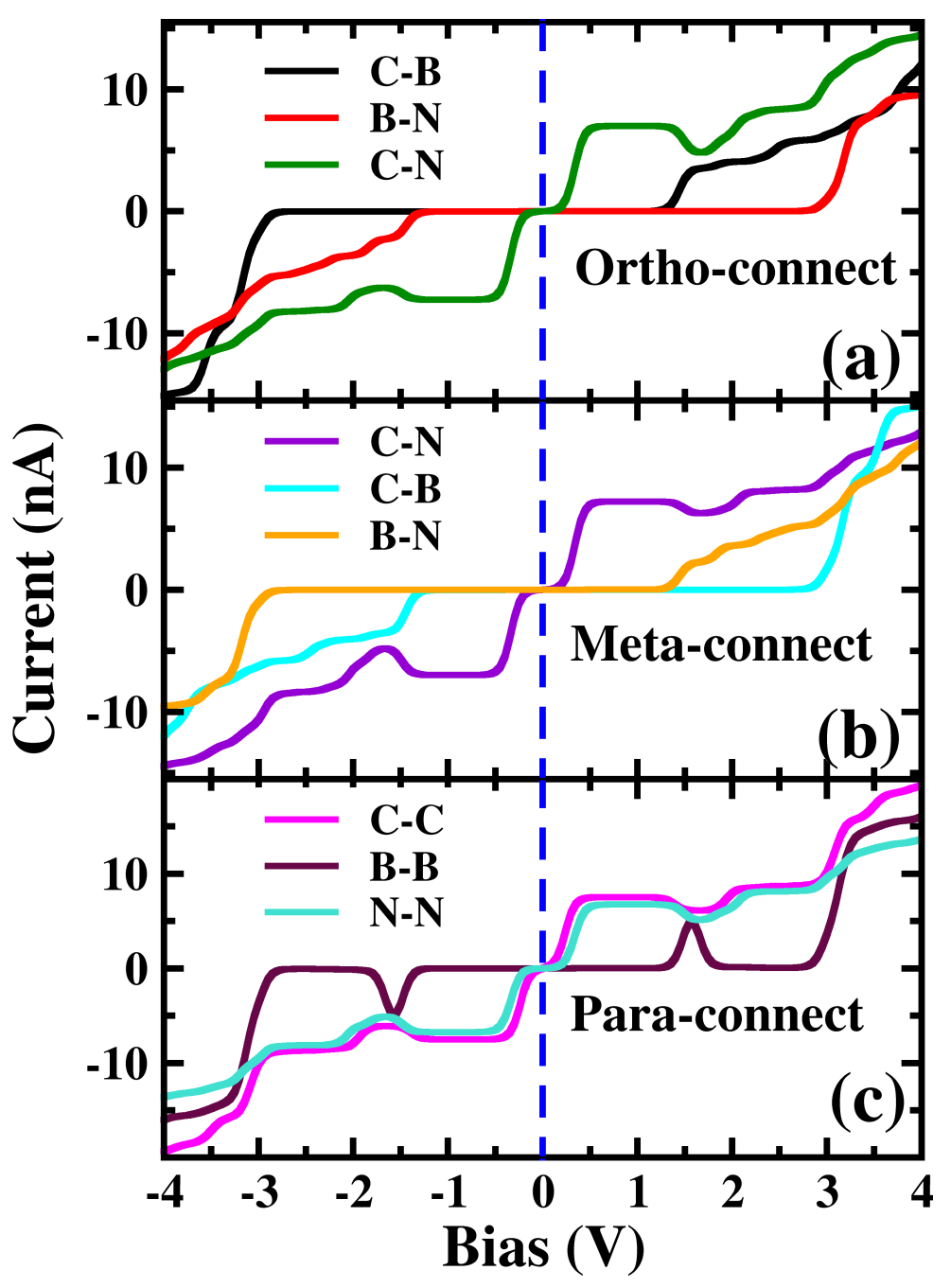

FIG. 2. Current (I) - source-drain voltage $\left(V_{S D}\right)$ characteristics of molecular device (bridge???) where electrodes are connected in (a) ortho, (b) meta and (c)para-position. Dashed blue line represents the $V_{S D}=0.0$. In the upper left insets of (a-c), the atomic sites where the electrodes are attached are shown.

steeply increases.

To find the reason behind the appearance of NDC in this molecular junction, we focus on the non-equilibrium occupation probabilities of transport-active many-body states and analyze how they get modified with the change in $V_{S D}$. As shown in Fig. 3 (b), at $0.0<$ $V_{S D}<0.2 \mathrm{~V}$, the 6 electron ground-state (i.e. $6 \mathrm{e}-\mathrm{gs}$ ) remains almost completely populated, leaving all other states nearly empty. In this bias region, the applied $V_{S D}$ is not enough to charge the molecule with an extra electron i.e. formation of anion is not favored. As the molecule is stable in neutral 6 electron state and there is no other eigenstate(s) within this energy range, there is no current through the molecular bridge. Consequently, the 
device remains in Coulomb blockade regime. As $V_{S D}$ increases, 7 electron ground-state (i.e. 7e-gs) becomes energetically accessible and there is transition of electrons from 6e-gs to 7e-gs, resulting in an increase in current (see Fig. 3 (a) and (b)). However, unlike other molecules explored previously 10 , the current due to this particular transition, appears to be very small, of the order of a few pA, as mentioned earlier. It simply indicates that 7e-gs is not suitable for electronic transport through the present molecular device. With further increase in $V_{S D}$ above a certain threshold bias-voltage, i.e. $V_{S D}>1.15 \mathrm{~V}, 7$ electron first excited state (7e-1st-es) becomes accessible. Consequently, the corresponding occupation probability starts increasing and exhibits sharp increase in the current for $V_{S D}>1.30 \mathrm{~V}$ (see Fig. 3 (a)). Importantly, here, the magnitude of current is of the order of nA, much higher than the current due to the tunneling from 6e-gs to 7e-gs. Interestingly, at $V_{S D}>1.55 \mathrm{~V}$ the probability of this conducting 7e-1st-es reduces, causing sharp drop in the current. In this particular bias-voltage, fundamentally, the transition from 7e-1st-es to 6 electron first excited states (6e-1st-es) becomes energetically possible. Thus, the net occupation probability of 7e1st-es starts reducing and consequently, for 6e-1st-es probability should increase. However, as the transition from 6e-1st-es to $7 \mathrm{e}-\mathrm{gs}$ is also probable at the same bias range, the net occupation probability of 7e-gs starts increasing as can be clearly seen in Fig. 3 (b). As 7e-gs are weakly conducting in nature, the increase in probability of this state results in the reduction of transporting current, exhibiting the NDC.

To understand the atomistic reason behind the different nature of 7 electron many-body states towards the electron transport in the sequential tunneling regime, we have analyzed the charge densities of these states. In Fig. 4, we represent the charge density of every sites of the molecule at their different low-energy transport active many-body states. As shown in Fig. 4, quite obviously, boron and nitrogen atoms in 6e-gs are electron deficient and rich, respectively, due to their intrinsic electronegativity. Interestingly, 7e-gs also has similar electron density distribution where the state remains largely polar due to charge localization at nitrogen and charge depletion at boron sites. Hence, when the electrodes are connected to two electron deficient boron atoms at para-position, transition of molecule from 6e-gs to 7e-gs at finite bias does not raise the electron-density at the electrode-molecule coupling region. And consequently, it results in very weak current flow through molecular junction. This explains the negligible current flow in the bias range of $0.0<V_{S D}<1.3 \mathrm{~V}$ and $1.85<V_{S D}<2.75 \mathrm{~V}$ in the junction as shown in Fig. 3 (a). On the other hand, in 

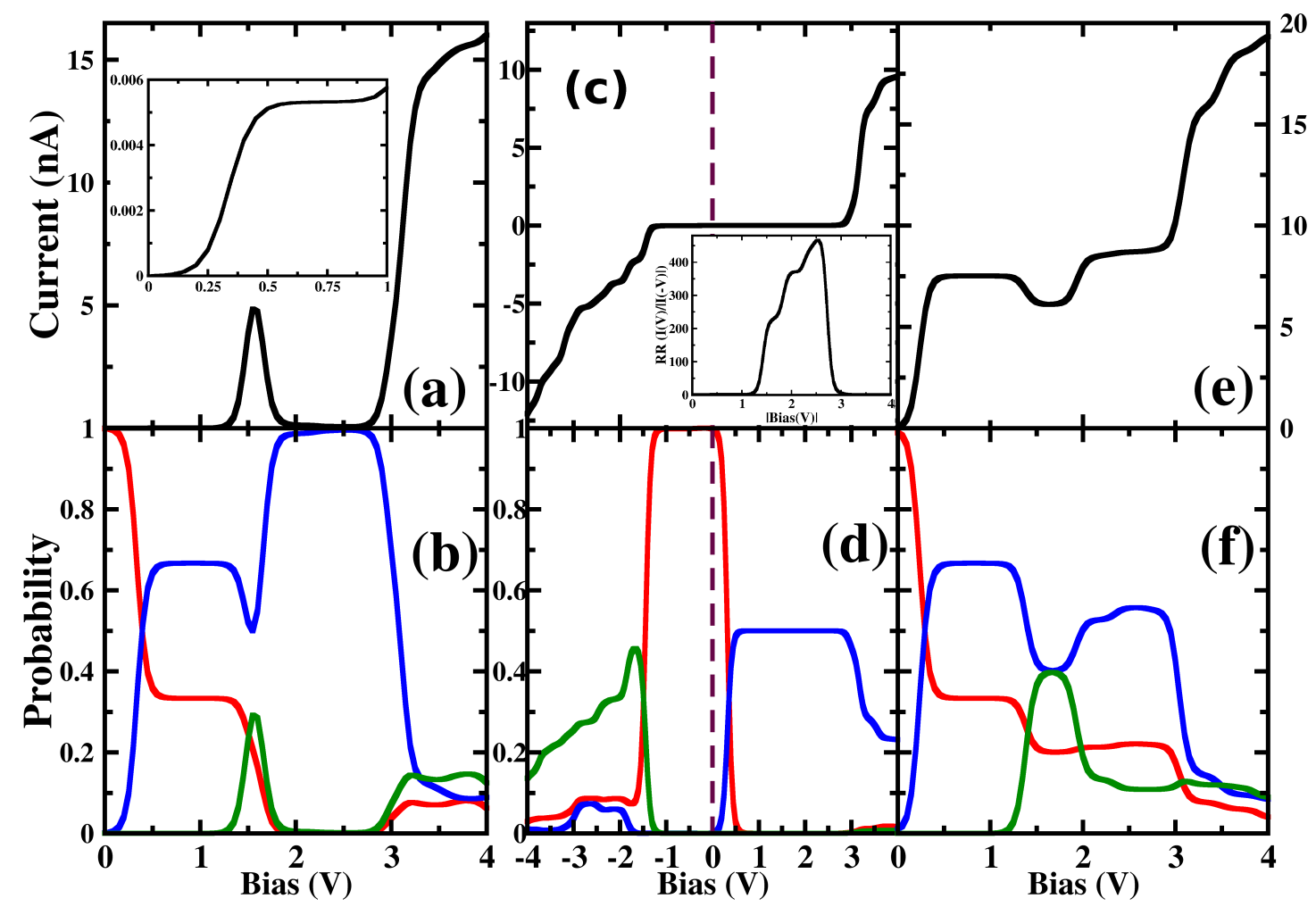

FIG. 3. The variation of current (I) with $V_{S D}$ for a device at different positions in benzene (top panel of the figure) and the occupation probabilities at the bottom panel of the figure for the same configuration. (a) I-V for two boron atoms at para position, (c) I-V for a boron and nitrogen atom at ortho position and (e) I-V for two carbons at para position. Occupation probabilities are represented by red line for $6 \mathrm{e}-\mathrm{gs}$, blue line for $7 \mathrm{e}$-gs and green line for $7 \mathrm{e}-1$ st-es states in all the figures. In the inset of (a), I-V plot for the junction connected by two borons has been given for bias-range $0.0<V_{S D}<1.0 \mathrm{~V}$.

the 7e-1st-es, electrons remain almost equally distributed over all the atomic sites. Thus, when the 7e-1st-es gets populated at particular bias, the electron densities at B atoms increase appreciably. And consequently, the current of magnitude of few $\mathrm{nA}$, flows through the molecular junction. This conduction of electron gets quenched when the higher bias (i.e. $\left.V_{S D}>1.55 \mathrm{~V}\right)$ populates the charge localized 7e-gs once again. At much higher bias i.e. $V_{S D}>2.80 \mathrm{~V}$ many more excited states start appearing as transport-active channels and consequently, the rapid growth of current can clearly be found in Fig. 3(a).

Thus, the population and depopulation of anionic ground state and 1st excited state of $\mathrm{B}_{2} \mathrm{C}_{2} \mathrm{~N}_{2} \mathrm{H}_{6}$ where charge distribution patterns are quite different and decisive for current flow, results in exciting non-linear behavior of current-voltage characteristics. 


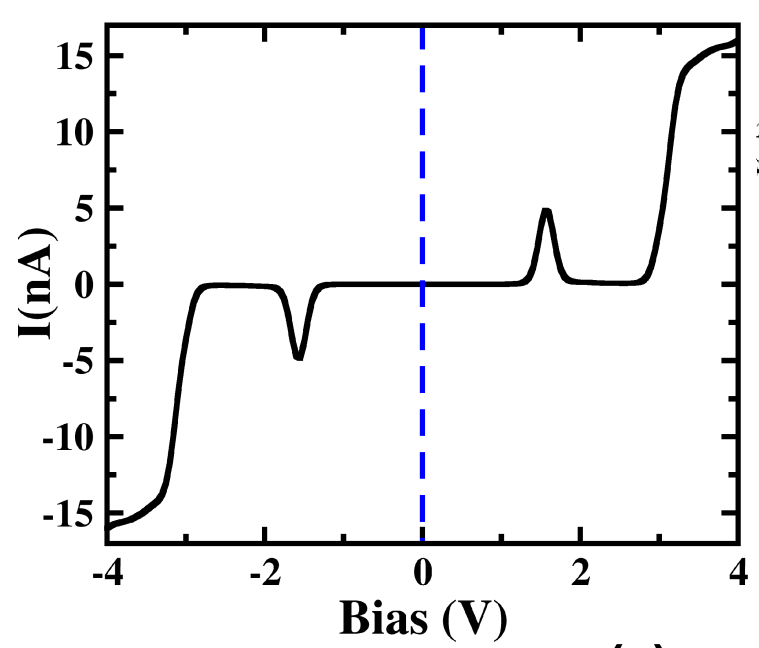

(a)

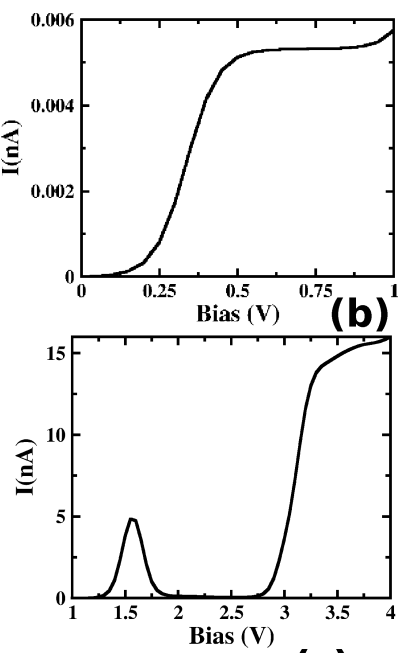

(c)

FIG. 4. The charge density distribution of the 6 electron ground state, 7 electron ground state and 7 electron 1st excited state over the sites of $\mathrm{B}_{2} \mathrm{C}_{2} \mathrm{~N}_{2} \mathrm{H}_{6}$. WRONG FIGURE

\section{B. Rectification}

Considering the device where electrodes are connected to chemically asymmetric ortho positioned $\mathrm{B}$ and $\mathrm{N}$ atoms, a prominent rectification of current appears in the I-V characteristics, as plotted in Fig. 3 (c). Although at positive bias regime, current of magnitude of a few nA flows through the device, the negative bias regime shows the appearance of nA current only after $V_{S D}=-2.80 \mathrm{~V}$. To have a quantitative measure about rectification, we further calculate rectification ratio $(\mathrm{RR})$ which is defined as the ratio between the absolute current-values at positive and negative voltage,

$$
R R(V)=|I(V) / I(-V)|
$$

We have plotted the rectification ratio in the inset of Fig. 3 (c). As can be seen, the $\mathrm{RR}(\mathrm{V})$ is as high as 466.2 at $2.55 \mathrm{~V}$. The occupation probability analysis of many-body states shows that at negative bias, major transition occurs between 6e-gs to 7e-1st-es, resulting in the flow of current at $V_{S D}<-1.25 \mathrm{~V}$ (see Fig. 3 (c, d)). In this negative bias region, the 7e-gs remain almost empty. On the other hand, on applying a positive bias of $V_{S D}>0.05 \mathrm{~V}$ to this molecular junction, occupation probability decreases for 6e-gs and increases for 7e-gs. However, the current remains negligible until $V_{S D}>2.80 \mathrm{~V}$. After this threshold voltage, 
current starts flowing due to the increase of occupation probability of energetically higher lying conducting states.

To find the fundamental reason for different conducting nature of these many-body anionic states, we look back at the charge density distribution per sites, as shown in Fig. 4 . It is evident that the boron site, attached with one of the electrode, is electron deficient for 7e-gs. Thus, the occupation probability of 7e-gs eventually gives rise to unfavorable electron transport through this molecular junction. However, for the 7e-1st-es, both the connecting sites i.e. $\mathrm{N}$ and $\mathrm{B}$ are electron rich in nature and consequently appears to be suitable for electron transport. So, different nature of occupation probability of these low-lying states at positive and negative bias, results in prominent rectification in the device. Note that, this kind of rectification also appears for other connectivities, where the atomic sites, connected to the electrodes, are chemically different, as can be seen in Fig. 2.

\section{Staircase}

In the following section, we focus on the molecular junctions where electrodes are connected to two carbon atoms which are in para-position to each other. As shown in Fig. 3 (e), the I-V characteristic of this particular device shows a step-like feature which is quite common for the junctions where sequential tunneling is the major mechanism for electron transport. As discussed previously, in this sequential tunneling regime, the charging energy for molecules is quite high and needs to be overcome by applying a certain $V_{S D}$. Before this threshold $V_{S D}$, we find Coulomb blockade regime in the device (see Fig. 3 (e)). For the present case, since we had chosen the Fermi level to be very close to the transition of 6 electron state to 7 electron state, the Coulomb blockade regime appears to be small. Further, as shown in Fig. 3 (f), with increment in $V_{S D}$, 7e-gs starts filling up and electrons start flowing through the junction. With increment in $V_{S D}$, other excited states of different charge and spin sectors start appearing in the active bias-window, which results in steps in the I-V plot for the molecular junction.

Importantly, for this particular device, as the electrodes are connected to two carbon atoms which are electron-rich in nature for $7 \mathrm{e}-\mathrm{gs}$, we find that electron flows through the molecular junctions, as increased $V_{S D}$ populates this anionic state. Small dip in the current for the bias range of $1.1 \mathrm{~V}<V_{S D}<2.0 \mathrm{~V}$ appears due to the finite occupation probability 
of 7e-1st-es which is less favorable towards electron transport in the present device. Lesser electron density at the carbon sites connected to electrodes, reduces the conducting nature of 7e-1st-es than that of 7e-gs.

Further, as shown in Fig. 2 (c), device with electrodes connected to para-positioned nitrogen atoms, also shows same kind of I-V characteristics.

\section{Effect of Magnetic Field}

In this section, we focus on the modulation of conductance of above mentioned molecular junction by applying a perpendicular magnetic field. The effect of the magnetic field with a wide range of strength has been investigated in this study. Firstly, we focus on the conformation where electrodes are connected to two B atoms at para-position. As shown in Fig. 5 (a), at relatively small magnetic field strength i.e. in the range of $0.001 \phi_{0}<$ $\phi<0.02 \phi_{0}$, the current increases at low bias $\left(0.15 \mathrm{~V}<V_{S D}<0.50 \mathrm{~V}\right)$ region with applied magnetic field strength. However, it is to be noted that the magnitude of resulting current remains in the pA order. The prominent NDC also remains almost unaltered for application of these relatively small magnetic fields. Interestingly, as we increase the magnetic field strength further, i.e. $0.02 \phi_{0}<\phi<0.10 \phi_{0}$ nature of current conduction starts changing at the NDC-peak region i.e. $1.0 \mathrm{~V}<V_{S D}<3.0 \mathrm{~V}$ (see Fig. 5 (c)). As shown in Fig. 6 (a) or (c)?????, applying magnetic field of $\phi>0.14 \phi_{0}$, the NDC-peak almost disappears and I-V exhibits staircase behavior. Thus, depending on the strength of magnetic field, we can modulate the transport characteristics of this molecular junction quite drastically.

To understand the modulation in I-V characteristics, we now concentrate on two mainly sensitive factors which get modified with applied magnetic fields- (1) the change in charge distribution on atomic-sites of the molecule and (2) occupation probabilities of transportrelevant six and seven electron many-body states.

On applying a relatively small magnetic field i.e. $0.001 \phi_{0}<\phi<0.02 \phi_{0}$, though the occupation probabilities of low-lying 6e and 7e-states remain almost unaltered, the charge distribution on the atomic sites get modified for them. Most importantly, as shown in Fig. 5 (b), B-sites which are highly electron deficient in the absence of magnetic field in 7e-gs hinders the current conduction and start getting populated with electrons with the application of the magnetic field. Charge density at B-sites for 7e-gs keeps on increasing 

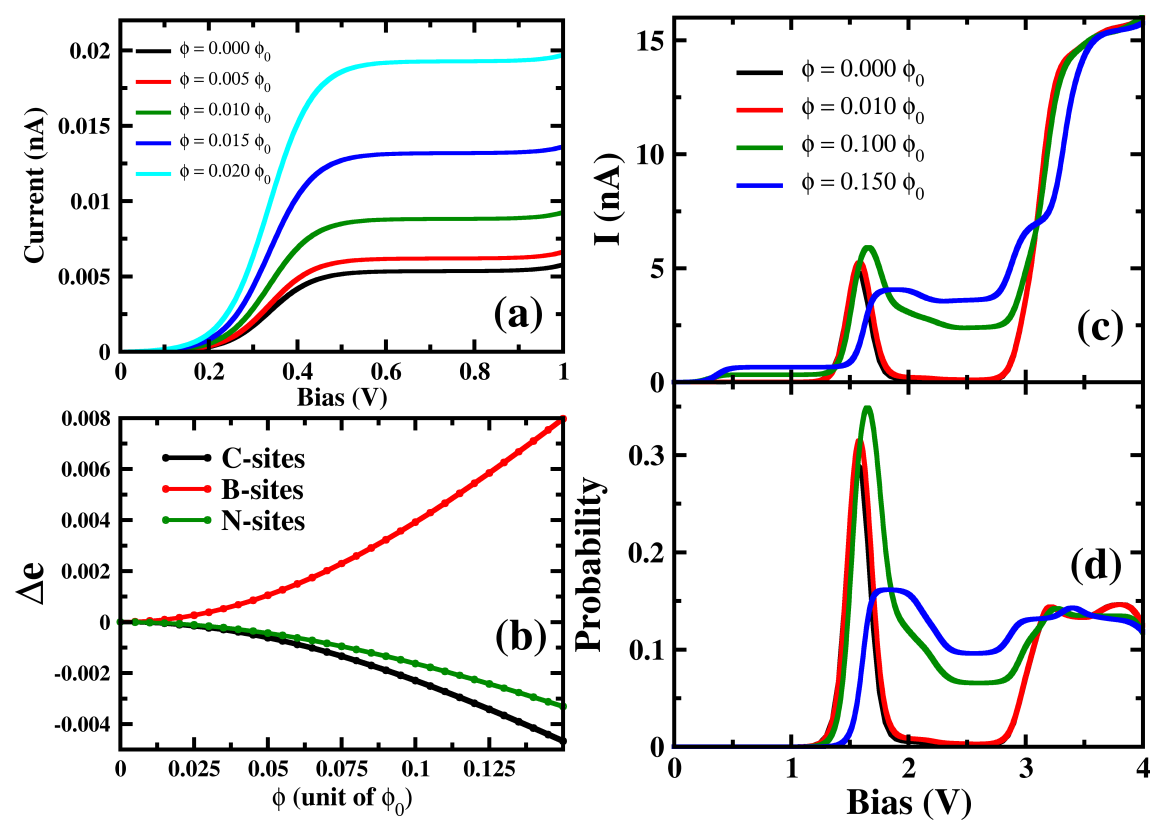

FIG. 5. (a) I-V characteristics for the low bias regime $\left(V_{S D}\right.$ of $\left.0 \mathrm{~V}<V_{S D}<1.0 \mathrm{~V}\right)$ versus magnetic field strength for para positioned boron connected device. (b) Charge density at boron sites with varying magnetic field strength. (c) I-V characteristics and (d) Occupation probabilities of 7e-1st-es for higher $V_{S D}$, for varying magnetic field strength.

as we increase the applied magnetic field strength. This increase of electron density on B-sites in turn makes 7e-gs more and more suitable for current conduction. Consequently, at low-bias i.e. $0.15 \mathrm{~V}<V_{S D}<0.50 \mathrm{~V}$ where 6e-gs to $7 \mathrm{e}$-gs transition occurs, amplitude of resulting current keeps on increasing with applied magnetic field strength. However, note that, at relatively small magnetic field, the increment of charge on B-atoms are small i.e. in the range of $2 \times 10^{-4} e^{-}$and the resulting current at low-bias remains in pA only (see Fig. 5). Thus, it is quite evident now that current through boron-connected molecular junction increases along with the accumulation of charge-densities of boron-sites.

As shown in Fig. 5 (c), with higher applied magnetic field i.e. $0.02 \phi_{0}<\phi<0.13 \phi_{0}$, where the prominent NDC peak gets affected, current does not completely switch-off at $V_{S D}>$ $1.80 \mathrm{~V}$. Here, we look into the atomic charge densities as well as occupation probabilities of relevant low-lying many-body states. As the B-sites of 7e-gs keeps on getting populated with application of stronger magnetic field, the current increases at the bias range of $0.15 \mathrm{~V}<$ $V_{S D}<0.50 \mathrm{~V}$ as discussed previously. More interestingly, it is apparent from Fig 5 (d) that though the occupation probability of highly conducting 7e-1st-es at $V_{S D}>1.55 \mathrm{~V}$ reduces, unlike previously, it remains finite at the bias range, $V_{S D}>1.80 \mathrm{~V}$. As this highly 


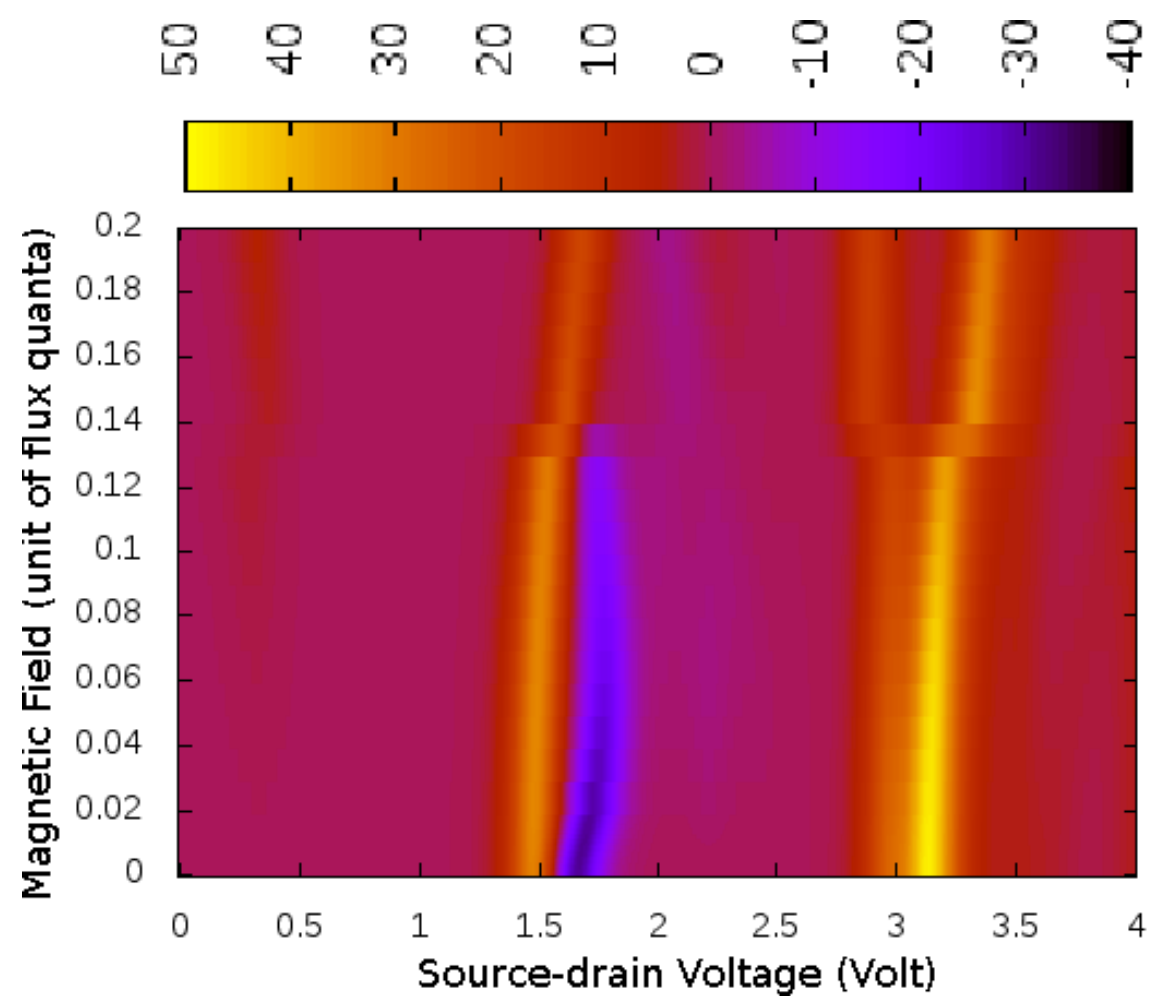

FIG. 6. The color map shows differential conductance $(\mathrm{dI} / \mathrm{dV})$ as a function of $V_{S D}$ and applied perpendicular magnetic field $(\phi)$ for para positioned boron connected device.

conducting state remains populated at the higher bias, the current never drops down to zero at any higher bias. Moreover, since 7e-gs is also quite conducting in nature, high occupation of this state also results in a measurable current flow through the molecular junction. Thus, at higher magnetic field strength, both charge densities at atomic sites as well as occupation probabilities of current-conducting states get modulated, resulting in change in the I-V characteristics.

When we apply higher magnetic field i.e. $\phi>0.14 \phi_{0}$, as shown in Fig 6, the NDC disappears completely and shows staircase behavior. Under these magnetic fields, at lowbias i.e. $V_{S D}>0.20 \mathrm{~V}$ the current gets switched-on and it keeps on increasing as the biasvoltage increases in a stepwise manner. From occupation probability, it is clear that current flow at low-bias is assisted by 6e-gs to 7e-gs transition, as mentioned earlier. Importantly, as the 7e-gs is now quite suitable for current conduction, at higher $V_{S D}$ where occupation probability of major conducting state 7e-1st-es reduces and that of 7e-gs increases, resulting in a stepwise behavior. Thus, there is no NDC in the I-V characteristics in the presence of higher magnetic field. 
Note that, generally it has been believed that magnetic flux needed to affect the molecular conduction is of the order of a magnetic flux quanta and required magnetic field is unrealistically huge in magnitude. However, as the bias dependent occupation probabilities and charge densities of transport active many-body states in our case are highly sensitive towards magnetic fields of much lower strength, we find evidence of modifications in I-V characteristic, even under a weak magnetic perturbation.

By analyzing the I-V characteristics of other molecule-electrode conformations, we find that magnetic field has very little effect on the I-V characteristics in these cases.

Interestingly, other perturbing factor, i.e. applied gate voltage, can also significantly affect the resulting current. Particularly, the molecular junction where prominent NDC character appears in the absence of gate voltage, exhibits significant alteration of net current flow upon the application of gate-voltage. As can be seen in Fig. 7, negative differential conducting nature can efficiently be switched off and on by varying the strength of the applied gate voltage. Note that, strength of the gate voltage, used in the present study, has been regularly achieved in experiments. Looking at the probability distribution, it becomes evident that as the strength of gate voltage increases, the probability of conducting 7e-1st-es around $1.0 \mathrm{~V}<V_{S D}<2.0 \mathrm{~V}$ gets diminished. Consequently, the NDC peak also disappears. Transition rates among the transport active many-body states (6e-gs to 7e-1st-es in present system) as well as their relative energies compared the electrodes fundamentally causes these kind of alteration in the $\mathrm{I}-\mathrm{V}$ characteristics.

\section{CONCLUSIONS}

To conclude, we have discussed the transport characteristics of cyclic molecular systems in sequential tunneling regime, applying well known kinetic equation approach. We chose our system to be a six-site molecule where donor, bridge and acceptor get arranged in a cyclic manner. Results clearly indicate that I-V character of presently investigated molecule strongly depend on the atomic nature of the sites, which are connected to the electrodes. When two different chemical species form the electrode-molecule coupling, prominent rectification of current appears. Importantly, when both B atoms are used as connecting sites, we find appearance of NDC in the device. All these exciting non-linear properties emerge due to the $V_{S D}$ dependent population and depopulation of low-lying excited states, which 


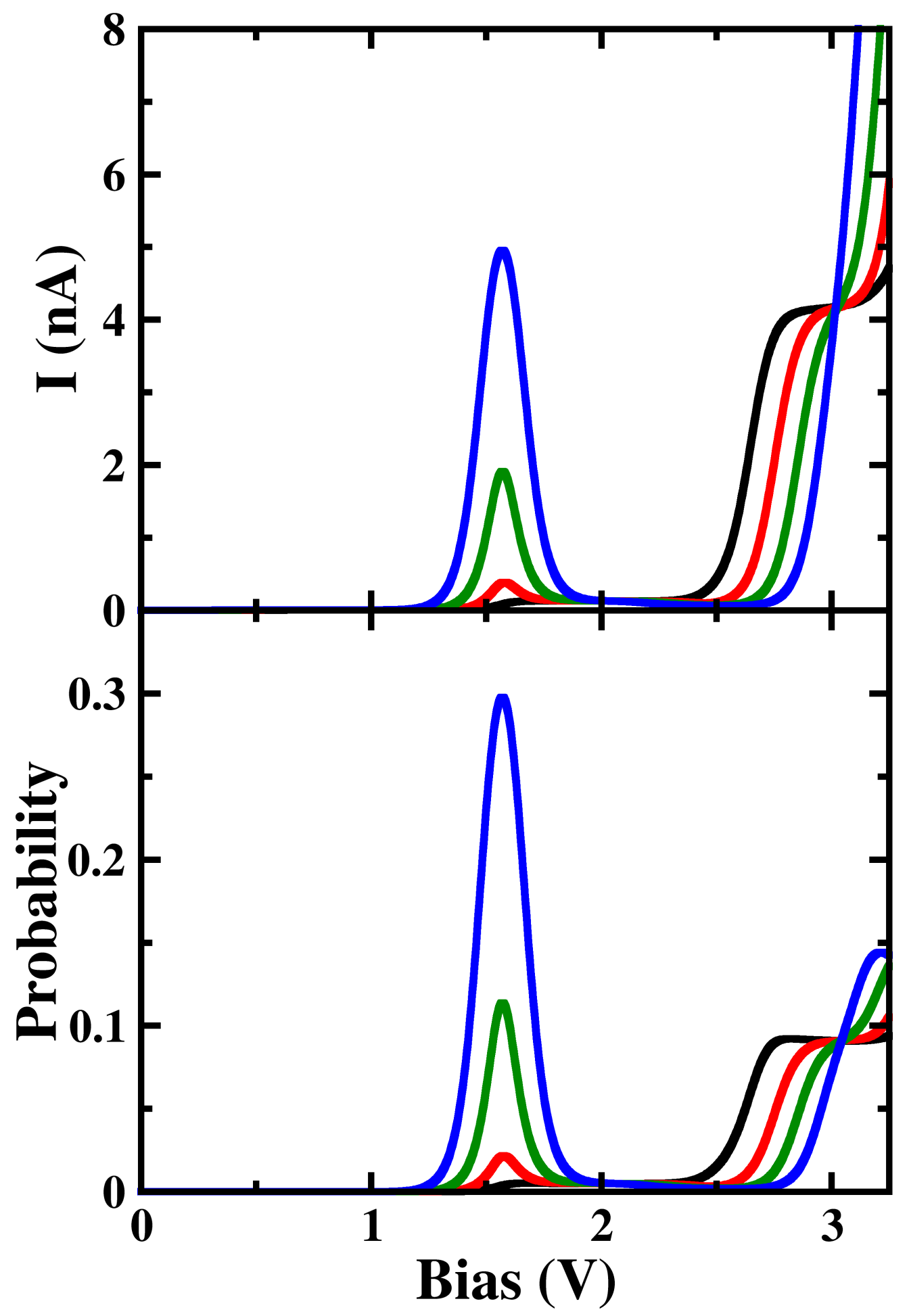

FIG. 7. Upper panel shows the I-V characteristic of boron-electrode connected junction at different gate voltage. Lower panel depicts the occupation probability of 7 e- 1 st-es at $0.0 \mathrm{~V}<V_{S D}<3.5 \mathrm{~V}$ where different gate voltage has been applied. Black, red, green and blue solid lines represent the current (upper panel) and probability (lower panel) with applied gate voltage of $0.15 \mathrm{~V}, 0.10 \mathrm{~V}$, $0.05 \mathrm{~V}$ and $0 \mathrm{~V}$, respectively. 
are quite different in terms of charge distribution pattern and hence nature of electron conduction. Fundamentally, the unequal charge distributions at different-sites of the molecule for these transport active many-body states determine the I-V characteristic. Particularly, the charge densities at the connecting sites to the electrode mainly control the current conduction through the molecular junction. Further, we also demonstrate the modulation of current conduction by applying perpendicular magnetic field. Depending on the strength, we find a number of interesting features, arising in the I-V characteristics. Thus, in this paper, we have successfully demonstrated various non-linear transport behavior appear for an intrinsically asymmetric heteronuclear cyclic molecule containing nano-junction.

* pati@jncasr.ac.in

1 J. R. Heath and M. A. Ratner, Physics Today 56, 43 (2003).

2 G. Cuniberti, G. Fagas, and K. Richter, Introducing molecular electronics: A brief overview (Springer, 2006).

3 J. L. Zhang, J. Q. Zhong, J. D. Lin, W. P. Hu, K. Wu, G. Q. Xu, A. T. Wee, and W. Chen, Chem. Soc. Rev. 44, 2998 (2015).

4 B. Xu and Y. Dubi, J. Phys.: Condens. Matter 27, 263202 (2015).

5 R. M. Metzger, Chem. Rev. 115, 5056 (2015).

6 A. R. Rocha, V. M. Garcia-Suarez, S. W. Bailey, C. J. Lambert, J. Ferrer, and S. Sanvito, Nat. Mater. 4, 335 (2005).

7 D. Aravena and E. Ruiz, J. Am. Chem. Soc. 134, 777 (2011).

8 Y. Dubi and M. Di Ventra, Rev. Mod. Phys. 83, 131 (2011).

9 J. Park, A. N. Pasupathy, J. I. Goldsmith, C. Chang, Y. Yaish, J. R. Petta, M. Rinkoski, J. P. Sethna, H. D. Abruña, P. L. McEuen, et al., Nature 417, 722 (2002).

10 M. Hettler, W. Wenzel, M. Wegewijs, and H. Schoeller, Phys. Rev. Lett. 90, 076805 (2003).

11 P. Parida, S. Lakshmi, and S. K. Pati, J. Phys.: Condens. Matter 21, 095301 (2009).

12 K. Ono and S. Tarucha, Phys. Rev. Lett. 92, 256803 (2004).

13 C. Caroli, R. Combescot, D. Lederer, P. Nozieres, and D. Saint-James, J. Phys. C 4, 2598 (1971).

14 A. Rocha and S. Sanvito, Phys. Rev. B 70, 094406 (2004). 
15 B. Muralidharan, A. Ghosh, and S. Datta, Phys. Rev. B 73, 155410 (2006).

16 B. Muralidharan and S. Datta, Phys. Rev. B 76, 035432 (2007).

17 B. Muralidharan, A. W. Ghosh, S. K. Pati, and S. Datta, IEEE Trans. Nanotechnol. 6, 536 (2007).

18 M. H. Hettler, H. Schoeller, and W. Wenzel, Europhys. Lett. 57, 571 (2002).

19 D. Darau, G. Begemann, A. Donarini, and M. Grifoni, Phys. Rev. B 79, 235404 (2009).

20 B. Song, D. A. Ryndyk, and G. Cuniberti, Phys. Rev. B 76, 045408 (2007).

21 Y. A. Pati and S. Ramasesha, J. Phys. Chem. A 118, 4048 (2014).

22 J. E. Sansonetti, W. Martin, and S. Young, Carbon 100, 1634 (2005).

23 E. Anderson, Z. Bai, C. Bischof, S. Blackford, J. Dongarra, J. Du Croz, A. Greenbaum, S. Hammarling, A. McKenney, and D. Sorensen, LAPACK Users' guide, Vol. 9 (Siam, 1999).

24 K. Wakabayashi, M. Fujita, H. Ajiki, and M. Sigrist, Physical Review B 59, 8271 (1999).

25 C. Rocha, A. Latgé, and L. Chico, Physical Review B 72, 085419 (2005). 